\title{
Avanços no diagnóstico do transtorno do humor bipolar
}

\author{
Igor Alcantara* \\ Ricardo Schmitt* \\ Alexandre Willi Schwarzthaupt* \\ Eduardo Chachamovich* \\ Miréia Fortes Vianna Sulzbach* \\ Rachel Tavares de Laforet Padilha* \\ Rafael Henriques Candiago* \\ Renato Moraes Lucas*
}

\section{INTRODUÇÃO}

Na última década assistimos a modificações na abordagem diagnóstica do Transtorno de Humor Bipolar. Inspirados em idéias da Grécia antiga e de Émile Kraepelin, muitos autores vêm propondo a expansão do conceito de bipolaridade para além dos critérios atualmente válidos.

Visando a abordar as formas atuais de pensar o Transtorno do Humor Bipolar (THB), realizamos uma revisão bibliográfica, buscando os seguintes aspectos: a) aspectos históricos do THB, b) a noção de Espectro Bipolar, c) os conceitos de Depressão Atípica (DeA) e Disforia Histeróide, d) os Estados Mistos, e) a relação entre THB e os Transtornos de Ansiedade, f)a relação com o diagnóstico de Transtorno de Personalidade Borderline (TPB), g) contraponto ao conceito de espectro bipolar.

\section{ASPECTOS HISTÓRICOS DO TRANSTORNO DO HUMOR BIPOLAR}

Loucura, melancolia, mania, fúria divina, possessão, bruxaria, tristeza, demência, psicose, depressão. Até chegarmos no Transtorno do Humor Bipolar, um grande caminho foi percorrido, que iniciou na Grécia e Roma juntamente com a história da nossa civilização. Descrever o seu trajeto é descrever a história da humanidade.

Os primeiros escritos sobre a existência da melancolia datam da civilização greco-romana e são descritas em personagens bíblicos como o rei Saul, no antigo testamento, e mitológicos, como na llíada, de Homero.

Saul, rei de Israel, um homem bravo, alto, forte e um valente guerreiro, luta contra os filisteus de maneira corajosa, mas em um dado momento, uma tristeza e um tormento tomam conta de sua vida. Não tem mais certeza de sua força e bravura, pensamentos de morte e tragédia passam a tomar conta dos seus dias, seus servos, preocupados, chamam a Davi, que tinha fama de tocar harpa e acalmar os estados de espírito; então, o atormentado rei se acalma. 
Saul melhora e tempos depois, volta a se sentir triste, desesperançoso e novamente atormentado. Não vendo saída para a sua situação, acaba por suicidar-se ${ }^{1}$.

Nos primeiros escritos médicos do século $V$, ainda havia muita influência da magia, fato que se modificou com os escritos naturalistas de Hipócrates ${ }^{1}$.

Hipócrates no século IV e $\mathrm{V}$ aC descrevia a melancolia (melan: negro; cholis: bile) como uma condição associada à aversão ao alimento, desalento, abatimento, insônia, irritabilidade e inquietude e afirmava que o medo ou a depressão prolongados significavam melancolia. Para ele, as doenças mentais seriam fenômenos derivados de um distúrbio humoral subjacente. Essa definição biológica que sobreviveu até o Renascimento fez parte da compreensão de que a saúde seria o equilíbrio dos quatro humores: sangue, bile amarela, bile negra e fleuma, e de que todas as doenças seriam produto do distúrbio desse equilíbrio $\mathrm{A}$ importância dessa teoria consiste na substituição da superstição pela biologia e na adoção do modelo de observação clínica como os componentes mais importantes para relatos de médicos e filósofos ${ }^{1,2}$.

Para Sorano de Éfeso, a mania envolvia prejuízo da razão com delírios, e a melancolia envolvia estar abatido, com propensão à raiva, quase nunca alegre e tranqüilo? .

Aretaeus da Capadócia, que viveu no século 1ํ dC, o "clínico da mania", foi o primeiro autor a sugerir que a mania é o estágio final da melancolia, uma visão que prevaleceu por séculos. Ele pensava que a mania e a melancolia tinham uma origem em comum na bile negra, em concordância com a teoria grega dos humores. Aretaeus também descreveu a ciclotimia. Suas observações são conhecidas hoje como o primeiro esforço nosológico em direção ao conceito moderno de transtorno bipolar².

Galeno de Pérgamo (128 a 201 d.C.), por sua vez, estabeleceu a melancolia como uma condição crônica e recorrente que poderia ser uma doença primária do cérebro ou secundária para outras doenças. Essa é uma elaboração mais abrangente da teoria humoral ${ }^{2}$.

Da Grécia clássica até o início da Idade Média, as afecções mentais e físicas eram cuidadas primariamente por médicos. À medida que essa função foi sendo delegada aos monastérios e religiosos, devido à queda do Império Romano, as idéias iniciais cederam lugar a um período de trevas. A doença mental passou a ser atribuída à magia, ao pecado e à possessão demoníaca, alvo da Santa Inquisição ${ }^{1,2}$.
No final da Idade Média (séculos XV e XVI), as ciências empíricas atraíam interesse e aceitação, estimuladas pela ascensão da filosofia metódica de Francis Bacon².

Pinel, em 1809, caracterizou a melancolia como uma doença composta de um número circunscrito de delírios em oposição à mania ou delírio generalizado que afetava todas as faculdades da mente.

Esquirol reconheceu o transtorno afetivo como uma forma distinta de perturbação mental, que ele chamou "lypemanie" (de perda, inibição e delírio mental) e abandonou o termo melancolia por considerá-lo excessivamente leigo e impróprio para uso técnico em medicina. Com seu trabalho, houve a transformação da visão de desestruturação psíquica global para o conceito de uma forma de loucura parcial, cujo distúrbio primário estaria nas emoções¹.

No início do século XIX, apesar da divulgação das idéias de Esquirol, a melancolia ainda era difundida como subtipo de mania, um distúrbio primário do intelecto, de intensidade excessiva de idéias e de natureza irreversível. A melancolia era vista, pois, como uma variante da mania ou como seu estágio evolutivo. Seu prognóstico era reservado, na medida em que a demência era vista como seu estágio final. Somente após adequar a descrição psicopatologica da depressão, esse conceito pôde ser reconhecido como integrante da noção de doença maníaco-depressivo ${ }^{1}$.

A palavra depressão derivou-se da medicina cardiovascular da época, por referir-se à redução da função; a palavra foi aplicada aos quadros mentais de forma análoga, como depressão mental, que representavam o rebaixamento do estado de espírito de pessoas que padeciam de alguma doença.

Na metade do século XIX, Jules Falret e J. $F$. Baillarger formularam a idéia de que mania e depressão representariam diferentes manifestações de uma única doença, essa corresponderia às primeiras concepções explícitas da doença maníaco-depressivo como entidade nosológica única².

O trabalho nosológico de Kraepelin foi decisivo para sintetizar os pensamentos de sua época e direcionar os estudos posteriores. Em 1899, ele agrupou todas as psicoses descritas anteriormente em uma entidade fundamental: doença maníaco-depressivo, que ele considerava uma afecção endógena e constitucional. Kraepelin foi o primeiro a desenvolver completamente um modelo de doença em psiquiatria, por meio de observações extensas e descrições cuidadosamente organizadas ${ }^{1,2,3}$. 
O ano de 1966 marca o renascimento da doença bipolar com duas das mais importantes publicações na área de transtorno do humor em toda a história da psiquiatria: os trabalhos de Jules Angst ("Sobre a Etiologia e a Nosologia de Psicoses Depressivas Endógenas") e de Carlo Perris, que publica "Um Estudo de Psicose Bipolar (Maníaco-Depressiva) e a Psicose Depressiva Recorrente Unipolar"3.

Dunner et alli, em 1976, introduziram a distinção entre Bipolar tipo1 (com episódios maníacos) e Bipolar tipo 2 (com episódios tipo hipomaníacos) ${ }^{3}$.

Iniciam-se as discussões sobre o Espectro Bipolar descritas ao longo deste trabalho.

\section{TRANSTORNO DO HUMOR BIPOLAR: UMA VISÃO DE ESPECTRO}

A noção de diagnóstico categorial para o Transtorno do Humor Bipolar utilizada nas classificações diagnósticas atuais (DSM-IV-TR e CID-10) vem recebendo críticas de alguns autores, sendo proposta uma abordagem dimensional para o diagnóstico destes transtornos.

A abordagem diagnóstica dimensional (continuum) propõe que a psiquiatria moderna promova uma mudança de suas diretrizes diagnósticas, inspirada nas idéias de Emil Kraepelin. Parte desta é representada pela noção de Espectro Bipolar, que se estenderia muito mais além das categorias diagnósticas de THB tipo I, THB tipo II e ciclotimia.

Esta mudança do paradigma de entendimento dos transtornos bipolares traz uma gama de conseqüências na abordagem e no tratamento do THB, incluindo um aumento de prevalência de $1 \%$ para cerca de $5 \%$ da população geral. Ademais, expande a utilização de estabilizadores de humor em detrimento da prescrição de antidepressivos, entre outros fármacos.

Representadas especialmente pelas publicações de Hagop Akiskal, polêmico autor na área, são propostas subdivisões específicas do THB em um número maior de subgrupos, descritas a seguir ${ }^{4,5}$ :

a) THB tipo I - Mania plena: caracterizada pela presença de uma síndrome maníaca clássica com sintomas psicóticos (mania significa psicose em Grego). Em alguns casos, o início do quadro pode apresentar uma mistura de depressão e mania, chamada de mania disfórica por alguns autores.

b) THB $I^{1 / 2}$ - depressão com mania prolongada, caracterizada pela presença de hipomania. Destaca que o limite que divide o quadro de so. Ainda assim, enfatiza que a hipomania não tem o caráter disruptivo presente na mania.

c) THB II - depressão com hipomania, caracterizada por episódios moderados ou severos de depressão intercalados com períodos de hipomania de, no mínimo, quatro dias de duração.

d) THB $\|^{1 / 2}$ - depressões ciclotímicas, caracterizadas pela presença de períodos de hipomania menores do que quatro dias em um paciente com episódios depressivos recorrentes. Destaca a importância desse subtipo, uma vez que a maioria dos episódios hipomaníacos tem de um a três dias de duração, não sendo assim considerados como tal pelos sistemas classificatórios.

e) THB III - hipomania associada a antidepressivos, caracterizada por pacientes que apresentam episódios de hipomania ou mania quando em uso de antidepressivos. Usualmente ocorre em pacientes com temperamento ciclotímico prévio.

f) THB III ${ }^{1 / 2}$ - bipolaridade mascarada ou desmascarada por abuso de estimulantes. Os autores propõem essa categoria para pacientes que apresentam episódios de hipomania ou mania diretamente associada ao uso de estimulantes.

g) THB IV - depressão hipertímica, caracterizada por pacientes que apresentam temperamento hipertímico de longa duração, com episódios depressivos tardios sobrepostos.

Os autores destacam que essas categorias ainda não são suficientes para descrever todas as características presentes no espectro bipolar. Assinalam também a importância do conceito de depressão pseudo-unipolar, dando ênfase à noção de que muitos pacientes diagnosticados como deprimidos possam apresentar na realidade alguma manifestação integrante do espectro bipolar.

\section{DEPRESSÃO ATÍPICA E DISFORIA HISTERÓIDE NO ESPECTRO BIPOLAR}

O conceito de Depressão Atípica (DeA) surgiu com o trabalho de West e Dally, em 1959, estudando pacientes responsivos e irresponsivos a IMAO (Inibidores da Mono-Amino-Oxidase) e notando a presença do histórico de fobias, de "conversões histéricas" e de ausências de sintomas neurovegetativos melancólicos entre os pacientes responsivos a IMAO ${ }^{6,7}$.

Desde então, temos até hoje divergências quanto à possibilidade da depressão atípica ser uma categoria diagnóstica única e constante. Diversos autores ${ }^{8-10} \mathrm{em}$ trabalhos atuais con- 
testam a validade dos atuais critérios do DSMIV-TR, principalmente quanto à permanência do Critério A (humor reativo) como necessário para validar os achados do Critério B (duas das seguintes: ganho de peso, hipersonia, paralisia "de chumbo" e sensibilidade à rejeição interpessoal.). Estes pacientes não podem ter critérios para melancolia ou depressão psicóticas (Critério C). ${ }^{7,10}$.

Com relação à pacientes do espectro bipolar, temos amplas variações de prevalência que decorrem da diversidade de metodologia empregada. Por exemplo, nos mais de 20 estudos relativos a espectro bipolar do italiano Franco Benazzi, suas amostras provêm de pacientes avaliados unicamente por ele, em sua clínica particular, escolhidos de forma não randomizada e sem controle. Desta série de casos, o autor publica diferentes análises. Muitos de seus trabalhos apresentam dados que foram aferidos de forma diversa dos critérios do DSMIV-TR. Assim sendo, seus trabalhos tendem a hiperestimar a prevalência de bipolares tipo II e de depressão atípica nesta população. Já no estudo de Rihmer e colaboradores, vemos a prevalência de bipolares tipo II muito baixa, provavelmente relacionada ao baixo valor preditivo da escala diangóstica "Diagnostic Interview Schedule" aferida por estudantes universitários treinados por uma semana. Como no exemplo do estudo de Rihmer ${ }^{11}$, há uma necessidade de desenvolver melhores métodos diagnósticos que aumentem o valor preditivo do diagnóstico de bipolares tipo II, podendo assim delimitar melhor quantos dos pacientes com DeA são do espectro unipolar e quantos são do espectro bipolar.

Em pacientes do espectro bipolar que têm episódio depressivo maior (EDM), pelo menos $30 \%$ apresentam características atípicas. Para Benazzi e Akiskal e colaboradores, estes índices podem chegar a mais de $60 \%$, variações decorrentes dos critérios utilizados para seleção e aferição das amostras ${ }^{6,12}$.

Em pacientes com diagnóstico de EDM, pelo menos $10 \%$ se tornarão bipolares ao longo da vida ${ }^{13}$.

A maioria dos poucos estudos que avaliam a frequência de espectro unipolar em pacientes com DeA fica em mais de $50 \%{ }^{13}$.

Outro grupo que merece consideração é aquele chamado de Disforia Histeróide. O termo Disforia Histeróide, apresentado por Klein e Davis, em 1968, é descrito como um subgrupo de depressão, que acomete especialmente muIheres e que apresenta sintomas afetivos atípicos associados a extrema intolerância à rejei- ção pessoal e vulnerabilidade à perda de relacionamentos românticos, aparentando comportamento histriônico. Ao serem desapontadas afetivamente, estas mulheres tornam-se agudamente deprimidas com intensa disforia, coléricas, hostis e com comportamento suicida, reagindo ainda, com frivolidade e/ou euforia em resposta a oportunidades amorosas. Os sintomas afetivos atípicos tendem a ser de hipersonia e hiperfagia, com especial consumo de doces e sentimento de estarem extremamente fatigadas. Liebowitz et al colocaram especial ênfase diagnóstica na reatividade do humor frente a eventos ou situações positivas ${ }^{14}$.

Himmelhoch et $\mathrm{al}^{15}$ referem-se à "depressão anérgica", que se caracteriza por severa fadiga, sintomas neuro-vegetativos inversos e retardo psicomotor e argumentam que esta apresentação é especialmente comum em pacientes bipolares. Este quadro sintomático é semelhante à descrição clássica da depressão atípica, com piora ao entardecer, insônia predominantemente inicial, hipersonia, aumento do apetite ou peso. Akiskal et $\mathrm{al}^{15}$ afirmam que a existência de sintomas atípicos em um quadro depressivo é um fator preditivo para o desenvolvimento de mania ou hipomania.

O DSM-IV-TR"12, ao incluir à "sensibilidade a rejeição interpessoal" aos sintomas atípicos de quadros depressivos, não diferencia Depressão atípica de disforia Histeróide como diagnósticos distintos. Benazzi ${ }^{16}$, estudando a prevalência daquele sintoma em pacientes bipolares tipo II e unipolares, tem com resultado uma maior freqüência do mesmo sintoma em Bipolares II, reforçando a idéia de que sintomas atípicos estejam mais relacionados a Transtorno do Humor Bipolar. Por outro lado, Posternak $^{15}$, estudando a prevalência de sintomas atípicos em patologias afetivas e ansiosas, observa uma taxa semelhante destes em unipolares e bipolares (I e II), ressaltando uma maior taxa para reatividade de humor entre unipolares e maior sensibilidade à rejeição entre bipolares.

Estes estudos são transversais, não permitindo aferir se o humor reativo ou a sensibilidade à percepção de rejeição interpessoal são primários ou secundários aos episódios de depressão atípica?

Apesar das limitações metodológicas, há uma forte tendência da literatura de encontrar mais pacientes com episódios de depressão atípica, que se apresentem com as seguintes características: prevalência maior no sexo feminino, idade de início precoce (adolescência ou início da vida adulta), maior cronicidade e 
menor remissão completa ${ }^{10}$ e mais comorbidades com transtornos de ansiedade como fobia social, transtorno do pânico (com agorafobia) $)^{8,17-19}$. Nos pacientes com comorbidade com transtornos de ansiedade, há uma maior taxa de tentativas de suicídio ${ }^{19}$. Alguns estudos têm achado maior prevalência de hipocondria e transtorno dismórfico corporal em pacientes com DeA ${ }^{8}$.

Uma maneira de resumir os achados atuais seria a formação de clusters fenomenológicos, ampliando o critério diagnóstico de características atípicas nas seguintes categorias não excludentes:

1. Pacientes em episódio depressivo maior com proeminência de sintomas de ansiedade, sem psicose com sintomas típicos de ansiedade psíquica, somática, tensão, ansiedade fóbica e pânico;

2. Pacientes com episódio depressivo maior com sintomas vegetativos atípicos como aumento do apetite, aumento da sonolência, sensação de peso nas pernas e/ou braços, associado a humor reativo e irritabilidade com padrão persistente de extrema sensibilidade à percepção de rejeição interpessoal;

3. Pacientes com episódio depressivo maior com características anérgicas (mais comumente encontrado em depressões bipolares).

Neste momento, temos pelo menos uma evidência mais clara para esta população: o uso de ADT (Antidepressivos Tricíclicos) não é eficaz nem efetivo, bem como aumenta as chances de viradas maníacas e ciclagem rápida em uma proporção significativa dos casos. Os IMAOs e ISRS (Inibidores Seletivos de Recaptação da Serotonina) apresentam eficácia aguda no manejo da DeA, tanto em unipolares quanto bipolares tipo II, não permitindo a remissão completa do quadro, mas diminuindo o prejuízo social e funcional, bem como frequência e intensidade de novos EDMs. A retirada da medicação provoca altas taxas de recaída nesta população, o que confirma que pacientes com residual sintomático tendem a ter maior risco de recaídas e cronicidade. Quando falamos de bipolares tipo II, os dados acima também se aplicam a eles, contudo os bipolares tipo II recebem ainda um risco aumentado de virada hipomaníaca, as quais são em geral três vezes mais elevadas que em unipolares. Isto ainda é mais grave quando vemos pacientes bipolares tipo I, sendo que nestes as taxas de virada maníaca são mais altas, a despeito de uma resposta mais errática aos antidepressivos. Do ponto de vista farmacológico, a lamotrigina vem sendo proposta como opção terapêutica, se não efetiva, em monoterapia que possa ser similar ao lítio em potencializar os efeitos dos antidepressivos atuais ${ }^{20-22}$.

\section{ESTADOS MISTOS}

A questão dos Estados Mistos não encontra consenso entre os pesquisadores. As idéias variam desde critérios mais rígidos como na CID-10 e no DSM-IV-TR (que requerem a presença de critérios para um episódio maníaco sobrepostos aos critérios de um episódio depressivo), até propostas mais abrangentes, incluindo todos os estados de mania que apresentem mínimos elementos depressivos, ou vice-versa.

A importância dos estados mistos é óbvia se considerarmos que, dependendo da classificação, entre 20 e $74 \%$ dos pacientes com doenças afetivas apresentaram em algum período um estado misto ${ }^{23}$.

O grupo de Akiskal, utilizando alguns conceitos de Kraepelin, sugere que esta classificação seja o resultado da interação entre os episódios depressivos maiores e maníacos com o temperamento prévio dos pacientes, nitidamente um temperamento oposto, resultando daí as diferentes apresentações. Assim, teríamos fundamentalmente 3 tipos de estados mistos ${ }^{24}$ :

Tipo 1: como resultante de um temperamento depressivo com a mania.Geralmente psicótico, com sintomas incongruentes com o humor.

Tipo 2: como resultante de um temperamento ciclotímico com uma depressão maior, no qual o substrato de temperamento ciclotímico permanece ativo durante o episódio depressivo, gerando sintomas como labilidade de humor, irritabilidade, fuga de idéias e abuso de substâncias, além de impulsividade sexual. Freqüentemente não psicótico, podendo ser confundido com Transtorno de Personalidade Borderline.

Tipo 3: como resultante de um temperamento hipertímico com uma depressão maior, na qual o episódio depressivo inclui apetite sexual, agitação e pressão para falar. São as depressões que evoluem mal com antidepressivo e geralmente respondem ao lítio.

Na Tabela 1 (Anexo 1), podem-se verificar os seis tipos de Estados Mistos propostos por Kraepelin resultantes da interação entre funções como humor, atividade e pensamento.

Os estados mistos são considerados desde Kraepelin as maiores evidências da relação entre mania e depressão. Este autor descreveu 


\section{ANEXO 1}

Tabela 1: Tipos de Estados Mistos propostos por Emil Kraepelin.

\begin{tabular}{llll}
\hline & Mania Ansiosa & Depressão agitada & $\begin{array}{l}\text { Mania com pobreza } \\
\text { de pensamentos }\end{array}$ \\
\hline Humor & Deprimido & Deprimido & Maníaco \\
\hline Atividade & Maníaco & Maníaco & Maníaco \\
\hline Pensamento & Maníaco & Deprimido & Deprimido \\
\hline & & & Mania Inibida \\
\hline Humor & Estupor Maníaco & $\begin{array}{l}\text { Depressão com } \\
\text { fuga de Idéias }\end{array}$ & Maníaco \\
\hline Atividade & Maníaco & Depressivo & Deprimido \\
\hline Pensamento & Deprimido & Deprimido & Maníaco \\
\hline
\end{tabular}

a mistura sindrômica destes dois pólos: tanto a entrada de sintomas depressivos dentro da mania, quanto o surgimento de hipomania em episódios depressivos completos ${ }^{25}$

Não existe uma terminologia definida para estes estados, sendo denominados de "estado misto", "mania mista", "depressão durante a mania" ou "mania disfórica", de maneira intercambiável na literatura. Apesar do conceito psicopatológico de Disforia incluir apenas a existência de irritabilidade ou ansiedade associada à tristeza ou exaltação afetiva, o DSM-IV-TR requer, para o diagnóstico de estado misto, manifestações depressivas ou de mania completas, apenas reduzindo o tempo necessário para o diagnóstico definitivo.

O número de critérios necessários para o diagnóstico permanece em discussão. González-Pinto ${ }^{26}$, referindo-se ao trabalho desenvolvido por S. McElroy em Ohio, USA, baseado nos critérios do DSM-IV-TR classifica a Mania Disfórica em Possível quando está presente apenas um sintoma depressivo, em Provável quando estão presentes dois sintomas depressivos e em Segura quando existem três sintomas.

Akiskal ${ }^{25}$, referindo-se à prevalência deste quadro dentro do THB como um todo, afirma que o número de sintomas depressivos dentro na mania ou vice-versa definiria a percentagem. Cita um trabalho realizado na França, envolvendo 100 pacientes (com sintomas maníacos pelo DSM-IV), avaliados por entrevista diagnóstica semi-estruturada derivada do DSMIV. Quando eram considerados os critérios de completa manifestação depressiva dentro da mania, foi verificada uma taxa de $6.7 \%$, mas quando foram avaliados pela inclusão de dois ou mais sintomas depressivos, a taxa verificada foi de $37 \%$ de maníacos disfóricos. O mesmo autor, estudando as taxas de estados mistos dentro de diferentes trabalhos, refere que em torno de $40 \%$ dos pacientes com THB apresentaram um evento misto no curso de suas doenças.

Akiskal ${ }^{25}$, realizando uma revisão a partir da denominação Mania disfórica, relata algumas características referidas na literatura: 1 abuso alcoólico e condições neuro-psiquiátricas seriam mais comuns dentro deste quadro; 2 - o mesmo tem sido mais bem caracterizado dentro de mulheres internadas; 3 - o curso da doença apresenta mais episódios depressivos que maníacos; 4 - tendência à repetição do quadro; 5 - história familiar de mais quadros depressivos que maníacos; 6 - grande potencial de suicídio; 7 - confusão e sintomas psicóticos, incluindo incongruência do humor, sendo consideradas importantes características clínicas. Estas características são igualmente ressaltadas por González-Pinto, bem como uma esperada recuperação mais lenta dos pacientes e um maior risco de recaída com maior freqüência de hospitalizações. Este autor acrescenta, acerca da idade de início da doença, que adolescentes estariam especialmente predispostos à mesma. Segundo Cassano ${ }^{27}$, a manifestação de THB em crianças abaixo dos 12 anos de idade é mais freqüentemente acompanhada de sintomas afetivos atípicos (disfóricos ou com ciclagem rápida), apesar de poderem apresentar manifestações maníacas ou depressivas semelhantes aos adultos. 
6. TRANSTORNO BIPOLAR E COMORBIDADE COM TRANSTORNOS ANSIOSOS

A associação entre sintomas ansiosos e depressão unipolar está bem estabelecida na literatura. Além das taxas de comorbidade dos transtornos de ansiedade com depressão serem bem conhecidas, atualmente já existem evidências de que esses transtornos dividem o mesmo substrato neurobiológico ${ }^{28,29}$.

No entanto, não podemos afirmar o mesmo para o transtorno bipolar. Classicamente, a literatura pouco contempla a relação entre essas duas entidades nosológicas. Comumente, na prática clínica diária, fazemos-nos as seguintes perguntas: a) qual a taxa de comorbidade entre transtornos ansiosos e transtorno bipolar? b) quais transtornos ansiosos são mais prevalentes em comorbidade?; c) a comorbidade piora o prognóstico e resposta ao tratamento? ; d) podemos predizer o aparecimento de sintomas bipolares em pacientes com transtornos de ansiedade?

A maioria dos estudos sobre essa comorbidade tem como ponto de partida pacientes com diagnóstico de transtorno bipolar; isto é, investigam as taxas de prevalência de transtornos ansiosos em pacientes com diagnóstico primário de THB. Dois grandes estudos populacionais ${ }^{15,29}$ - Epidemiologic Catchment Area (ECA) e National Comorbidity Survey (NCS) - demonstraram taxas de prevalência ao longo da vida (lifetime) de transtornos ansiosos significativamente mais altas entre pacientes bipolares quando comparados à população em geral. $\mathrm{O}$ estudo ECA demonstrou que pacientes com transtorno bipolar apresentam prevalência de $21 \%$ para transtorno do pânico e Transtorno Obsessivo-Compulsivo (TOC), comparados a $0,8 \%$ e $2,6 \%$ para a população em geral. No estudo NCS, a prevalência de transtornos ansiosos em geral no THB foi de 92,9\% contra $24,9 \%$ da população. Confirmando esses achados, estudos clínicos com amostras menores reproduzem esses números. Um estudo conduzido por Angst ${ }^{30}$ demonstrou significativa associação entre hipomania e transtorno do pânico e fobia social. Tais achados têm levado à especulação de que a prevalência de sintomas de ansiedade em pacientes com THB possa ser até mesmo maior do que a encontrada em pacientes com depressão unipolar ${ }^{31,32}$. Outro achado interessante é que cerca de $43 \%$ dos pacientes com mania mista apresentam algum episódio bem definido de ataque de pânico (29), o que corrobora as observações de Kraepelin sobre sintomas de humor e ansiedade. Tam- bém algumas evidências apontam que sintomas de ansiedade são um marcador do aparecimento de sintomas depressivos na mania ${ }^{33,34}$.

A investigação do diagnóstico comórbido específico de ansiedade demonstra os seguintes achados:

1 - Transtorno do Pânico: em pacientes com diagnóstico de THB, a prevalência de pânico varia de $20,8 \%$ a $33,1 \%$. Para pacientes com depressão unipolar, essa taxa é de $10 \%$. Pacientes com THB têm um risco 1,8 vez maior de apresentar pânico quando comparados à depressão unipolar. Além disso, um estudo de história familiar ${ }^{35}$ demonstrou que cerca de $88 \%$ dos pacientes com transtorno do pânico tinham ao menos um familiar com história de THB.

2 - TOC: o estudo ECA demonstrou prevalência de $21 \%$ em pacientes com THB, comparado a $12,2 \%$ em pacientes com depressão unipolar. Além disso, um estudo clínico ${ }^{36}$ sugeriu que os sintomas de TOC podem variar durante o curso do THB. Por exemplo, os sintomas de TOC podem desaparecer nos períodos de mania e reaparecer durante a remissão ou em episódios depressivos.

3 - Fobia social: o estudo NCS demonstrou prevalência de $47,2 \%$, comparada a $13,3 \%$ na população em geral ${ }^{29}$.

Embora com consideráveis limitações metodológicas, esses estudos vêm demonstrando que a comorbidade entre transtornos de ansiedade e THB piora consideravelmente o prognóstico desses pacientes, assim como já evidenciado nos casos de ansiedade e depressão unipolar. Pacientes com THB e algum outro transtorno ansioso apresentam risco aumentado de suicídio, abuso de substâncias psicoativas e resposta pobre ao lítio ${ }^{37,38}$. Evidências de grau $\mathrm{C}$ apontam o valproato de sódio como boa alternativa para tratamento farmacológico desses pacientes ${ }^{29}$.

A investigação longitudinal de pacientes com transtorno de ansiedade para detectar prevalência de THB não é muito descrita na literatura até hoje. Alguns estudos apontam prevalência de $13,5 \%$ a $23,1 \%$ de THB em pacientes diagnosticados primariamente com pânico. Para pacientes com TOC, essa taxa foi de cerca de $15 \%$ e de $21,1 \%$ para fobia social ${ }^{29}$

O momento atual é de grande efervescência no que se refere a critérios diagnósticos e apresentação clínica do THB. Inúmeras publicações e encontros científicos vêm acontecendo tanto no nosso meio, quanto em outros centros mundiais. A relação entre ansiedade e THB é apenas um dos tantos aspectos contemplados nessa grande discussão. Podemos afirmar 
que o momento é muito mais de criação de hipóteses e campos de pesquisa do que exatamente de respostas. No entanto, algumas "certezas provisórias" podem ser destacadas: a) a comorbidade entre ansiedade e THB existe; b) a presença de um dos diagnósticos parece aumentar o risco de uma apresentação comórbida; c) as evidências atuais apontam para uma prevalência aumentada de pânico, TOC e fobia social em pacientes com THB; d) comorbidade piora prognóstico e dificulta o tratamento farmacológico; e) talvez, a melhor conclusão que podemos tirar das evidências atuais é que devemos ficar atentos para a associação entre essas duas doenças e suas conseqüências na apresentação clínica, prognóstico e tratamento escolhido.

\section{TRANSTORNO DO HUMOR BIPOLAR E TRANSTORNO DE PERSONALIDADE BORDERLINE}

Uma importante discussão acerca das apresentações clínicas do THB diz respeito ao seu relacionamento com os Transtornos da Personalidade, em especial o Transtorno da Personalidade Boderline (TPB).

A relação entre Personalidade e Transtornos de Eixo I em geral foi proposta por Goodwin e Jamison em quatro diferentes níveis ${ }^{39}$ :

a) Personalidade como fator predisponente para doença.

b) Personalidade como uma manifestação da doença.

c) Personalidade como um modificador da doença. ença.

d) Personalidade sendo alterada pela do-

Falando-se exclusivamente sobre a relação entre THB e TPB, são apresentadas as seguintes possibilidades de relacionamento entre os dois diagnósticos ${ }^{5}$.

a) Os dois representam diferentes pontos no espectro de uma mesma entidade nosológica.

b) Representam diferentes doenças com sintomatologia semelhante.

c) Um diagnóstico representa apenas um subgrupo do outro.

d) Um deve ser encarado como fator causal ou contribuinte para o outro.

Importantes sintomas, como instabilidade afetiva, impulsividade e momentos psicóticos, são apresentados tanto por pacientes com THB, como por aqueles com TPB. Este achado sugere que apenas a classificação psiquiátrica atual não apresenta a chave para a solução do pro- blema $^{40}$.

Vários aspectos são estudados na tentativa de elucidar a melhor forma de diagnosticar os dois transtornos: estudos de sono, história familiar, avaliação de resposta farmacológica, avaliação do padrão de relações interpessoais, uso de questionários padronizados e discussão de casos clínicos por especialistas.

A avaliação do padrão de sono demonstrou uma diminuição na latência do sono REM (Rapid Eyes Movement) para bipolares e borderlines em três estudos, sugerindo compartilharem o mesmo diagnóstico. No entanto, os pacientes considerados Borderlines também apresentavam histórico de Depressão Unipolar ou Bipolar. A latência do sono REM não distinguiu entre borderlines e bipolares ou entre borderlines e controles normais ${ }^{41}$.

Avaliação da história familiar não esclarece em definitivo a questão. Akiskal et al relatam aumento de bipolaridade entre familiares de borderlines ${ }^{42}$; no entanto, Pope et al encontraram apenas depressão unipolar. Neste estudo, os pacientes borderlines também apresentavam depressão ${ }^{43}$.

O uso de moduladores de humor em pacientes com TPB produz melhoras nos sintomas de humor, mas não alcança uma total modificação no curso da doença, como costuma fazer nos transtornos bipolares. Existe ainda a confusão pelo fato do TPB poder se apresentar em comorbidade com um transtorno de humor, e apenas este último responder ao tratamento ${ }^{44,45}$.

Os instrumentos diagnósticos costumam ser bastante limitados para diagnosticar Transtornos de Personalidade. O TCI (Temperament e Caracter Inventory), desenvolvido por Cloninger, foi capaz de distinguir entre Bipolares e Borderlines. Por sua vez, o grupo de Akiskal et al desenvolveu o "Inventário de Temperamento Bipolar", que realizou diagnóstico positivo para THB em uma amostra de pacientes com $\mathrm{TPB}^{39,46}$.

Quando foi avaliado o padrão de relacionamento interpessoal, comparando THB e TPB, verificou-se que paciente borderlines podem ser diferenciados dos bipolares. Borderlines tendem a vivenciar seus relacionamentos como hostis e com risco de abandono. Bipolares tendem a encarar os outros como submissos e sob seu controle ${ }^{47}$.

Rudra Prakash ${ }^{48}$ lembra que, por vezes, diagnósticos de TPB têm sido baseados na irresponsividade a tentativas medicamentosas. Contudo, a responsividade a antiepiléticos não significa necessariamente que dado paciente 
possua um diagnóstico de THB, haja vista a presença de problemas neurológicos sub-clínicos em pacientes com TPB.

Em resumo, a discussão acerca da validade diagnóstica do TPB, ou sua inclusão no espectro dos Transtornos do Humor, está polarizada, entre dois grupos. Akiskal et al., através das semelhanças fenomenológicas, fatores biológicos e achados de história familiar, defendem a inclusão do TPB no espectro do THB. Outros autores, avaliando as relações interpessoais, diferenças fenomenológicas e resposta ao tratamento, consideram que o Transtorno de Personalidade Borderline é um diagnóstico válido e diferente do THB.

\section{CONTRAPONTOS À IDÉIA DE ESPECTRO BIPOLAR}

Guy Goodwin ${ }^{49}$ externa sua preocupação com a ampliação do termo "hipomania", observando que este redimensionamento de significados psicopatológicos tem sido publicado em trabalhos que contam com amostragens pequenas. O termo hipomania estaria sendo utilizado como representante de uma generalização que relaxa nos critérios do DSM-IV-TR para severidade e tempo. A isso, acrescenta a questão de quando se deve ou não tratar a elevação do humor. Observa que, no Reino Unido, a disfunção social e ocupacional pode estar presente ou ausente quando se realiza um diagnóstico de hipomania. Assim, contrário ao uso generalizado do termo, defende o uso do DSM-IV-TR e seus critérios específicos de classificação, como orientador de condutas terapêuticas ${ }^{49}$.

Angst \& Marneros ${ }^{1}$ levantam problemas concernentes ao conceito de espectro bipolar, por exemplo, quando apontam a falta de estudos que sustentem o subtipo "bipolar III", observando não haver provas advindas de estudos controlados com placebo e somente estudos prospectivos indicando que depressões com hipomania, primeiramente manifestadas com o uso de antidepressivos, freqüentemente progridem para transtorno bipolar. Ainda aponta o fato de estudos familiares não suportarem amplamente o conceito de espectro bipolar. Assim, propõem que o conceito de espectro restrinja-se à sintomatologia observada na clínica e não evolua pra conclusões na questão genéti$\mathrm{ca}^{1}$.

Diagnósticos de Transtornos da Personalidade ficam preteridos, muitas vezes, por treinamentos inadequados nesta área, sem valorização de aspectos de funcionamento social, desenvolvimental e dados da história do pa- ciente $^{50}$. Feiner acrescenta que o uso da expressão transtorno bipolar "resistente" ao tratamento, fala de farmacoterapias, sem significar que intervenções não-biológicas também hajam sido oferecidas. Ainda diz que transtornos bipolares estariam tão superdiagnosticados quanto a esquizofrenia o foi no passado, e que o diagnóstico de transtorno bipolar está ameaçado de diluição (com o conceito de espectro), assim como ocorreu no passado, até que Kraepelin estabelecesse as diferenças entre "dementia praecox" (esquizofrenia) e "manic depressive insanity" (transtorno bipolar).

\section{CONCLUSÃO}

Avaliar a ocorrência de THB na população é fundamental, devido aos enormes prejuízos causados por este transtorno. A idéia de espectro bipolar contribui para que menos pacientes sejam inadvertidamente medicados com antidepressivos, piorando o curso de suas doenças. No entanto, é necessária cautela para evitar-se a diluição do conceito com a conseqüente perda dos princípios fundamentais do atendimento médico, a saber: diagnóstico e tratamento.

O THB apresenta-se em alta comorbidade e piora de forma inequívoca o curso e a resposta ao tratamento dos transtornos de ansiedade.

Os quadros de depressão atípica, disforia histeróide, episódios mistos e o transtorno de personalidade borderline carecem de estudos de validade diagnóstica. Por outro lado, apenas a avaliação de respostas farmacológicas não é suficiente para equacionar este problema.

O amplo debate nos mostra que, talvez, um dos pontos mais frágeis da Psiquiatria seja o do diagnóstico. O fenômeno é conhecido desde antes da Grécia Antiga, mas a dificuldade em defini-lo se apresenta cada vez mais atual. Considerar o diagnóstico do THB como diferencial parece ser uma conduta prudente e adequada na prática clínica.

\section{REFERÊNCIAS BIBLIOGRÁFICAS}

1. Angst J, Marneros A. Bipolarity from ancient to modern times: conception, birth and rebirth. Journal of Affective Disordes 67, 2001; 3-19.

2. Cordás TA . Depressão: da bile negra aos neurotransmissores - uma introdução histórica. Editorial Lemos, 2002.

3. Moreno RA, Moreno D H. Transtorno Bipolar do Humor. Editorial Lemos, 2002, p 11-61.

4. Akiskal H, Pinto O. The Evoking Bipolar Spectrum: Prototypes I, II, III and IV. Psych Clin of North America, vol 22, n 3:517-34, 1999 . 
5. Goodwin FK, Jamison KR: Manic Depressive Ilness. Oxford University Press, 1990.

6. Kaplan \& Sadock's Comprehensive Textbook of Psychiatry. $7^{\circ} \mathrm{Ed}$. Lippincott Williams and Wilkins 2000; USA

7. Parker G, Roy K, Mitchell P, Wilhelm K, Malhi D, HadziPavlovic D. Atypical depression: a reappraisal. Am J Psychiatry 2002; 159: 1470-1479.

8. Posternak MA, Zimmerman M. Partial validation of the atypical features subtype of major depressive disorder. Arch General Psychiatry 2002; 59: 70-76.

9. Angst J, Gamma A, Sellaro R, Zhang H, Merikangas K Toward validation of atypical depression in the community: results of the Zurich cohort study. J Affect Disord 2002; 72: 125-138.

10. American Psychiatry Association. Manual Diagnóstico e estatístico de transtornos mentais $4^{\underline{a}}$ ed rev. Ed. Artmed, Porto Alegre, Brasil, 2002.

11. Rihmer Z, Szádóczky E, Füredi J, Kiss K, Papp Z. Anxiety disorders in bipolar I, bipolar II and unipolar major depression: results from a population-based study in Hungary. J Affect Disorder 2001; 67: 175-179.

12. Sotsky SM, Simmens SJ. Pharmacotherapy response and diagnostic validity in atypical depression. J Affect Disord 1999; 54: 237-247.

13. Zubieta JK, Pande AC, Demitrack MA, Two years followup of atypical depression. J Psych Research 1999;33:23 29.

14. Peruggi G, Akiskal HS, Lattanzi L, Cecconi D, Mastrocinque C, Patronelli A, Vignoli S, Bemi E. The high prevalen ce of "soft" bipolar (II) features in atypical depression. Comprehensive Psychiatry 1998; 39: 63-71

15. Posternak MA, Zimmerman M, The prevalence of atypical features across mood, anxiety and personality disorders. Comprehensive Psychiatry 2002; 43: 253-262

16. Benazzi F. Exploring aspects of DSM-IV-TR interpersonal sensitivity in bipolar II. Journal of Affect. Disor. ,60, 43-46, 2000

17. Levitan RD, Lesage A, Parikh SV. Reversed neurovegetative symptoms of depression: a community study of Ontario. Am J Psychiatry 1997; 154: 934-940.

18. Horwath E, Johnson J, Weissman MM. The validity of major depressive with atypical features based on a community study. J Affect Disord 1992; 26: 117-126.

19. Benazzi, F. Prevalence of bipolar II disorder in outpatient depression: a 203-case study in private practice. J Affect Disord 1997; 43(2): 163-166.

20. Sachs GS, Printzs DJ, Kahn DA, Carpenter D, Docherty JP. The expert consensus guideline series: Medication treatment of bipolar disorder. Postgrad Med Special Report 2000; April: 1-104

21. Frye MA, Ketter TA, Kimbrell TA, Dunn RT, Speer AM, Osuch EA. A placebo-controlled study of lamotrigine and gabapentine monotherapy in refractory mood disorders. $J$ Clin Psychopharmacol 2000; 20: 607-614.

22. Zevaj-Lacombe S, Tabarsi BA. Lamotrigine: a review of clinical studies in bipolar disorder. Can J Psychiatry 2001; 46: 328-333

23. Marneros A: Expanding the group of bipolar disorders. Journal of Affective Disorders 62(2001) 39-44.

24. Akiskal HS The distinctive mixed states of bipolar I, I and III .Clin. Neuropharm 15(1) 632-33.

25. Akiskal HS, Bourgeois ML, Angst J, Post R, Möller H-J and Hirschfeld. Re-evaluating the prevalence of and diagnostic composition within the broad clinical spectrum of bipolar disorders. J. Aff. Disor. 59,2000;5-30.

26. González-Pinto A e Lalaguna B. La Mania Disfórica. In: E Vieta. Transtornos Bipolares - Avances clínicos y terapêuticos. 2001.

27. Cassano GB, McElroy SL, Brady K, Nolen WA and Placi- di GF. Current issues in the identification and management of bipolar spectrum disorders in "special populations". J.Aff. Disor. 59,2000; 69-79

28. Angst $\mathrm{J} \&$ Vollrath $M$. The natural history of anxiety disorders. Acta Psychiatr Scand 1991;84:446-452.

29. Freeman MP, Freeman SA, McElroy SL. The comorbidity of bipolar and anxiety disorders: prevalence, psychobiology and treatment issues. J. Affective Disorders 68, 2002; 1-23.

30. Angst J. The emerging epidemiology of hypomania and bipolar II disorder. J.Affect.Disord., 1998; 61-65.

31. Chen YW, Dilsaver SC. Comorbidity of panic disorder in bipolar ilness: evidence from the epidemiologic catchment area survey. Am J Psych, 1995; 280-282.

32. Chen, YW; Dilsaver, SC. Comorbidity for obsessive-compulsive disorder in bipolar and unipolar disorders. Psych Res, 1995; 57-64.

33. Cassidy F, Forest K, Murry E, Carrol BJ. A factor analysis of the signs and symptoms of mania. Arch Gen Psych, 1998; 27-32.

34. Cassidy F, Forest K, Murry E, Carrol BJ. Signs and symptoms of mania in pure and mixed episodes. J Affect Disord, 1998; 187-201.

35. Mc Kinnon DF, Xu J, McMahon FJ, Simpson SG, Stine OC, McInnis MG, DePaulo JR. Bipolar disorder and panic disorder in families: an analysis of chromossome 18 data. Am J Psych, 1998; 155: 829-831.

36. Perugi G, Akiskal HS, Pfanner C, Presta S et al. The clinical impact of bipolar and unipolar affective comorbidity on obsessive-compulsive disorder. J Affect Disord, 1997; 46:15-23.

37. Yong LT, Cooke RG, Robb JC, Levitt AJ, Joffe RT. Anxious and non-anxious bipolar disorder. J Affect Disord, 1993;29:49-52.

38. Feske V, Frank E, Mallinger AG, Fagiolini A, Shear MK et al. Aanxiety as a correlate of response to the acute treatment of bipolar I disorder. Am J Psych, 2000;157:956962.

39. Deltito J, Martin L, Riefkhl J, Austria B, Kissilenko A, Corless P, Morse C. Do patients with borderline personality disorder belong to the bipolar spectrum? J Affect Disord 2001; 67: 221-228.

40. BlackerD et al. Contested Boundaries of Bipolar Disorders and the Limits of Categorical Diagnosis in Psychiatry. Am J Psychiatry 1992; 149:1473-1483.

41. Benson, KL; King, R; Gordon, D et al. Sleep Paterns in Borderline Personality Disorder. Journal of Affective Disorders 1990; 18: 267-273.

42. Akiskal HS, Chen SE, Davis GC et al. Borderline: an adjective in search of a noun. J Clin Psychiatry 1985; 46:41-48.

43. Pope HG, Jonas JM, Hudson JI et al. The validity of DSMIII Borderline Personality Disorder: a Phenomenologic, family History Treatment Response, and Long-term Follow-up Study. Arch Gen Psychiatry 1983; 40:23-30.

44. Frankenburg FR, Zanarini MC. Divalproex Sodium Treatment of Women with Borderline Personality Disorder and Bipolar-II Disorder: A Double -Blind Placebo-Controlled Pilot Study. J Clin Psychiatry 2002; 63 442-446.

45. Henry C, Mitropoulou V, New AS, Koenigsberg HW, Silverman J, Siever LJ. Affective instability and impulsivity in borderline personality and bipolar II disorders: similarities and differences. J Psychiatr Res, Nov-Dec 2001; 35(6): 307-312

46. Atrevaidya N, Hussain SM. Borderline Personality Disorder and Bipolar Mood Disorder: Two Distinct Disorders or a Continuum? J Nerv Ment Dis 1999; 187(5): 313-5.

47. Benjamin LS, Wonderlich SA. Social Perceptions and Borderline Personality Disorders: The Relation to Mood 
Disorders. Journal of Abnormal Psychology 1994; 103(4); 610-624.

48. Prakash R, Roback HB. (1997) Letters to the editor. Am J Psychiatry, Aug; 154(8): 1176-1177.

49. Goodwin, G. Hypomania: what's in a name? Britsh Journal of Psychiatry, 2002; 181: 94-95.

50. Feiner FN. (1997) Letters to the editor Am J Psychiatry, Aug; 154(8):1176.

\section{RESUMO}

Atualmente, vemos transformações no diagnóstico do Transtorno de Humor Bipolar (THB). A prática clínica exige conhecimento mais detalhado da correlação THB - outras doenças psiquiátricas.

Nessa revisão não-sistemática, foram abordados aspectos diagnósticos do THB: a) histórico, b) Espectro Bipolar, c) Depressão Atípica (DeA) e Disforia Histeróide, d) Estados Mistos, e) relação THBTranstornos de Ansiedade, f) relação com o diagnóstico de Transtorno de Personalidade Borderline (TPB), g) contraponto ao conceito de espectro bipolar.

A doença é conhecida desde a Grécia Antiga. Os estudos baseados nas publicações de Hagop Akiskal expandem o diagnóstico para além dos critérios usualmente utilizados, criando o conceito de espectro bipolar. A alta prevalência de comorbidade entre THB e Transtornos de Ansiedade corroboram que ambos compartilham o mesmo substrato neurobiológico. O debate demonstra que não há consenso, expondo a fragilidade dos nossos métodos diagnósticos. Entretanto, a revisão mostra a utilidade de sempre considerar o THB como diagnóstico diferencial.

Descritores: Transtorno do humor bipolar, Diagnóstico, Critérios, Comorbidade.

\section{ABSTRACT}

Nowadays, the diagnostic criteria for bipolar disorder (BD) have been changed. We need a better awareness about $B D$-another psychiatry diseases correlation for a good clinical practice.

The authors conducted a non-systematic review about some diagnostic topics in BD: a) history, b) bipolar spectrum, c) atypical depression and hysterical disforia, d) mixed states, e) $B D$ and anxious disorders comorbidity, f) $B D$ and borderline personality disorder relationship, g) bipolar spectrum contest.

The $B D$ has been investigated since Hypocrites time. The studies based on Akiskal's ideas put the BD diagnostic beyond the common diagnostics criteria, establishing a new entity called bipolar spectrum. The high prevalence of comorbidity between $B D$ and anxious disorders perhaps confirm the evidence that they share a same neurobiological substratum. There is no consensus and we realize that our diagnostics methods are fragile. However, this review point that the $B D$ must be considered a differential diagnostic in clinical practice.

Keywords: Bipolar disorder, diagnostic, criteria, comorbidity.

Title: Advances in the diagnosis of bipolar mood disorder

Endereço para correspondência:

Igor Alcantara

Rua Ramiro Barcelos, 1954 apto. 605

90035-002 - Porto Alegre - RS

E-mail: igoralcantara@uol.com.br

Copyright (c) Revista de Psiquiatria do Rio Grande do Sul - SPRS 